Non-peer reviewed EarthArXiv preprint

\title{
Vertical slowness constrained joint VTI parameters and event \\ locations inversion for microseismic monitoring
}

\author{
Congcong Yuan, Song Han, Jie Zhang \\ Geophysical Research Institute, University of Science and Technology of China, \\ Hefei, Anhui 230026, P. R. China. \\ CongcongYuan:congcy@mail.ustc.edu.cn \\ Song Han: hansong8@mai.ustc.edu.cn \\ Jie Zhang (Corresponding author): jzhang25@mai.ustc.edu.cn
}




\section{ABSTRACT}

The construction of accurate anisotropic velocity models for the monitoring region plays a very important role in microseismic monitoring. Due to ignorance of anisotropy, serious distortions may happen in the results of microseismic location and interpretation. Several effective methods have been developed to invert for VTI parameters and event locations using microseismic traveltime, while the results have a strong dependent on the initial velocity model and enough ray coverage due to strong nonlinearity of the problem. Meanwhile, microseismic waveform inversion for anisotropic parameters is still challenging because of low signal-noise ratio (SNR) of the data and high computation cost. Based on the assumption of small horizontal velocity variation, we develop a method jointly inverting event locations and velocity updates using traveltimes and vertical slowness estimates. The vertical slowness estimates are independent of the source information and easy to be obtained. We apply the method and currently developed method into four synthetic examples under different circumstances, the comparison demonstrates that our method is more stable than the other one, especially under the poor coverage. In addition, the field data test indicates that our proposed approach constrains the event locations better comparable to the other method.

Key words: microseismic traveltime, anisotropic parameters, event locations, slowness method. 


\section{Non-peer reviewed EarthArXiv preprint}

\section{INTRODUCTION}

Including information about anisotropy is essential for the location of microseismic events during the hydraulic fracturing, e.g., in gas/oil shale cases where strong VTI anisotropy (up to 30\%) is commonly seen (Warpinski et al., 2009; Eisner et al., 2011; Li, 2013; Zhang et al., 2015). Due to only vertical sound speed or velocity known from the sonic logs or calibrated by perforation shots, the method becomes urgent and demanding to construct an effective anisotropic model.

Several recipes have been successively developed to solve this problem. Mah and Schmitt (2003) use a global search method to simultaneously determine all elastic moduli of a homogenous composite material from travel times. However, the computer time required by their method increases very rapidly with the number of observations and unknown parameters. To obtain a velocity model that better reflects the structure between the actual microseismicity and receivers, Grechka et al. (2011) simultaneously estimate the general anisotropy of the medium while locating the microseismicity, assuming the medium is a homogeneous anisotropic space. However, the receiver array often spans a large depth range and is likely to be in a very different formation from where the microseismic events are located. As a result, it could be unrealistic to assume the medium to be homogeneous in these cases. Li et al. (2013) propose a new anisotropy velocity inversion method to determine the transversely anisotropic structure and relative event locations using double-difference with back azimuth constraint. Han et al. (2015) simultaneously determine event locations and Thmosen's parameters in VTI 


\section{Non-peer reviewed EarthArXiv preprint}

medium using quasi-P and SH-waves. However, if $\mathrm{P}$ - and $\mathrm{S}$-wave vertical velocities extracted from the logging data are not accurate enough, and raypath coverage is poor, the trade-off between anisotropic and event parameters should be a concern.

To avoid the limitation above, we ought to provide the inversion enough accurate initial anisotropic parameters or extra efficient information to constrain the inversion. Several methods modeling anisotropic velocities have been developed using dipole sonic logs (Walsh et al., 2008; Erwemi et al, 2010; Woerpel, 2010). Although effective are these methods, in microseismic processing they need at least two wells. The requirement cannot be met in only one well treatment. Additionally, the Thomsen's anisotropy parameters measured in the lab are utilized to constrain the anisotropic inversion in (Li, et al., 2013; 2014). It is known that we are usually difficult to obtain these parameters in the lab measurement. Furthermore, Michel and Tsvankin (2016) utilize several synthetic cases to show the accuracy of the anisotropic waveform inversion for microseismic velocity analysis and event location. As we all know that it is difficult to apply the waveform inversion into the real cases due to some factors, such as its high computation demanding and poor quality of the real data. Hence, a faster and easier solution to the issue, proposed here, is to use phase slowness components or polarizations of quasi-P, quasi-SV, and $\mathrm{SH}$ waves to constrain the original traveltime inversion.

The idea of applying the slowness or polarization method for estimating local anisotropy from VSP is hardly novel. Local estimation of the anisotropy parameters from traveltimes and polarizations recorded in boreholes is discussed in a number of 


\section{Non-peer reviewed EarthArXiv preprint}

publications (Miller and Spencer, 1994; Horne and leaney, 2000; Pevzner et al, 2001; Dewangan and Grechka, 2003; Grechka and Mateeva, 2007; Grechka et al., 2007; Rusmanugroho and McMechan, 2012a,b; Tamimi et al, 2015). In addition, Asgharzadeh et al., (2015) reveal that if the assumption of lateral homogeneity is valid, quasi-P wave slowness method is a robust method for VTI parameters. Hence, the estimations of local parameters using slowness method can provide reliable initial models or constraint for the traveltime inversion. To simplify the processing steps, we incorporate the slowness method into the conventional traveltime inversion and extend the inversion to the vertical slowness constrained inversion method.

In this study, we simplify the $3 \mathrm{D}$ problem to $2 \mathrm{D}$ by projecting all of the events onto a vertical $(\mathrm{XZ})$ plane, with a vertical $\mathrm{Z}$-axis and a horizontal $\mathrm{X}$-axis representing the distance to the monitoring well, considering the symmetric feature of the 1D velocity model and vertical monitoring well. In the field data, we will project the results back to 3D using the event azimuths, which are determined from separate analysis of the calibrated P-wave polarizations. In the framework of our work, we first introduce the traveltime inversion method and modified inversion method. Then we apply both methods to four synthetic cases and one field case to analyze their validity upon the recovery of model parameters and event location.

\section{Theory}

In this section, we introduce two inversion methods used for the model construction or both model construction as well as event locations. The first one is the currently 
developed inversion method based on the arrival times, then inspired by the idea of the slowness method (Miller and Spencer, 1994), we propose our method by the combination of the extended slowness method.

The synthetic traveltimes play an important part in the traveltime inversion. Based on the slowness method (Miller and Spencer, 1994; Kim, 1999), phase dispersion relations for $\mathrm{qP}, \mathrm{qSv}$, and $\mathrm{SH}$ waves can be derived from Kelvin-Christoffel equation. If the horizontal phase slowness components $P_{X}$ are given, then the vertical phase slowness components can be obtained,

For quasi-P and quasi-Sv waves, the vertical slowness components are expressed as,

$$
\begin{aligned}
& B=\frac{1}{\alpha_{0}^{2}}+\frac{1}{\beta_{0}^{2}}-2\left(1+\delta+(\varepsilon-\delta) \frac{\alpha_{0}^{2}}{\beta_{0}^{2}}\right) P_{X}^{2} \\
& C=\left((1+2 \varepsilon) P_{X}^{2}-\frac{1}{\alpha_{0}^{2}}\right)\left(P_{X}^{2}-\frac{1}{\beta_{0}^{2}}\right) \\
& P_{Z}^{q P}=\sqrt{\frac{B-\sqrt{B^{2}-4 C}}{2}} \\
& P_{Z}^{q S v}=-\sqrt{\frac{B-\sqrt{B^{2}-4 C}}{2}}
\end{aligned}
$$

For SH wave, the vertical slowness components are expressed as,

$$
P_{Z}^{S H}=\sqrt{\frac{1}{\beta_{0}^{2}}-(1+2 \gamma) P_{X}^{2}}
$$

where $P_{Z}^{q P}, P_{Z}^{q S v}$, and $P_{Z}^{S H}$ are the vertical slowness components of quasi-P and quasi-Sv and SH waves, respectively. $\alpha_{0}, \beta_{0}, \varepsilon, \delta$, and $\gamma$ are the Thomsen's parameters defined in (Thomsen, 1986). Then based on the relations of slowness components and group velocities, the traveltime can be calculated. Here, we synthesize the traveltimes with the shooting algorithm by trying different $P_{X}$ (Han and Zhang, 2015). 


\section{Traveltime inversion method (TM)}

In microseismic data processing, the observed arrival time $T_{i j}$ from a microseismic event $i$ to a receiver $j$ installed in the well is formulated as,

$$
\begin{aligned}
& T_{i j}=\tau_{i}+t_{i j} \\
& t_{i j}=\int_{\text {raypath }} \frac{1}{V_{g}} d l
\end{aligned}
$$

where $T_{i j}$ is the observed arrival time. $\tau_{i}$ is the origin time of the event $i . t_{i j}$ is the traveltime from a microseismic event $i$ to a receiver $j . V_{g}$ is the group velocity structure. $d l$ is an element of raypath length. The event location, origin time, and group velocity structure are the unknowns. The traveltimes can be synthetized by the raytracing method of the shooting algorithm that based on the slowness components (Cerveny, 2005; Tang and Li, 2008; Han and Zhang, 2015).

Based on the prior information, the linear approximation of the arrival time residuals can be expressed to the perturbations of the event and group velocity model parameters,

$$
\Delta T_{i j}=T_{i j}^{o b s}-T_{i j}^{c a l}=\Delta \tau_{i}+\sum_{k=1}^{3} \frac{\partial T_{i j}}{\partial x_{k}} \Delta x_{k}+\sum_{l=1}^{\text {raypath }} \frac{\partial T_{i j}}{\partial V_{g}^{l}} \Delta V_{g}^{l}
$$

where $T_{i j}^{o b s}$ is the observed arrival time data. $T_{i j}^{c a l}$ represents the calculated arrival time data based on the prior information. $\Delta \tau$ is the origin time perturbation. $\Delta x$ stands for the event location perturbation.

According to the relations between the group velocity and Thomsen's parameters (Thomsen, 1986; Kim, 1999; Han, 2016), we can transform the equation (5) into the following equations, 
For quasi-P wave, the above equation can be written as,

$$
\begin{aligned}
\Delta T_{i j}^{q P}= & \Delta \tau_{i}+\sum_{k=1}^{3} \frac{\partial T_{i j}^{q P}}{\partial x_{k}} \Delta x_{k}+\sum_{l=1}^{\text {raypath }} \frac{\partial T_{i j}^{q P}}{\partial \alpha_{0}^{l}} \Delta \alpha_{0}^{l}+\sum_{l=1}^{\text {raypath }} \frac{\partial T_{i j}^{q P}}{\partial \beta_{0}^{l}} \Delta \beta_{0}^{l} \\
& +\sum_{l=1}^{\text {raypath }} \frac{\partial T_{i j}^{q P}}{\partial \varepsilon^{l}} \Delta \varepsilon^{l}+\sum_{l=1}^{\text {raypath }} \frac{\partial T_{i j}^{q P}}{\partial \delta^{l}} \Delta \delta^{l}
\end{aligned}
$$

For quasi-Sv wave, the above equation can be written as,

$$
\begin{aligned}
\Delta T_{i j}^{q S v}= & \Delta \tau_{i}+\sum_{k=1}^{3} \frac{\partial T_{i j}^{q S v}}{\partial x_{k}} \Delta x_{k}+\sum_{l=1}^{\text {raypath }} \frac{\partial T_{i j}^{q S v}}{\partial \alpha_{0}^{l}} \Delta \alpha_{0}^{l}+\sum_{l=1}^{\text {raypath }} \frac{\partial T_{i j}^{q S v}}{\partial \beta_{0}^{l}} \Delta \beta_{0}^{l} \\
& +\sum_{l=1}^{\text {raypath }} \frac{\partial T_{i j}^{q S v}}{\partial \varepsilon^{l}} \Delta \varepsilon^{l}+\sum_{l=1}^{\text {raypath }} \frac{\partial T_{i j}^{q S v}}{\partial \delta^{l}} \Delta \delta^{l}
\end{aligned}
$$

For SH wave, the above equation can be written as,

$$
\Delta T_{i j}^{S H}=\Delta \tau_{i}+\sum_{k=1}^{3} \frac{\partial T_{i j}^{S H}}{\partial x_{k}} \Delta x_{k}+\sum_{l=1}^{\text {raypath }} \frac{\partial T_{i j}^{S H}}{\partial \beta_{0}^{l}} \Delta \beta_{0}^{l}+\sum_{l=1}^{\text {raypath }} \frac{\partial T_{i j}^{S H}}{\partial \gamma^{l}} \Delta \gamma^{l}
$$

where $\Delta \alpha_{0}, \Delta \beta_{0}, \Delta \varepsilon, \Delta \delta$, and $\Delta \gamma$ are the perturbations of the traveltime to the Thomsen's parameters. To express simply, we include all the Thomsen's parameters into the model vector $m$. Due to the complexity of detecting arrival time for quasi-SV wave, and the multi-value of quasi-SV group velocities, we are difficult to adopt the traveltime information of quasi-SV wave in reality, although we can compute the quasiSV traveltimes in means of shortest raypath method by avoiding shadow zone. Hence, only quasi-P and SH traveltimes are utilized in this study and the scheme adopted for the inversion for event locations and velocity model is therefore constructed as,

$$
\left(\begin{array}{ccc}
1 & W_{m} A_{m}^{q P} & W_{x} A_{x}^{q P} \\
1 & W_{m} A_{m}^{S H} & W_{x} A_{x}^{S H}
\end{array}\right)\left(\begin{array}{c}
\Delta t_{0} \\
\Delta m / W_{m} \\
\Delta x / W_{x}
\end{array}\right)=\left(\begin{array}{c}
T_{o b s}^{q P}-T_{s y n}^{q P}-T_{0}^{q P} \\
T_{o b s}^{S H}-T_{s y n}^{S H}-T_{0}^{S H}
\end{array}\right)
$$

where $A_{m}$ and $A_{x}$ are the derivatives of traveltime with respect to Thomsen parameters and event locations, respectively. $\Delta m, \Delta t_{0}$, and $\Delta x$ represent the 


\section{Non-peer reviewed EarthArXiv preprint}

perturbations to Thomsen parameters, origin time, and event location, respectively. $T_{o b s}^{p} \quad$ and $T_{o b s}^{s h}$ are the observed arrival times of quasi-P and SH waves, respectively. $T_{s y n}^{p}$ and $T_{s y n}^{s h}$ mean the synthetic traveltimes of quasi-P and SH waves, respectively. The initial values will be set before the inversion, then in every iteration the values will be updated. In order to reduce the influence of different magnitude of Thomsen parameters and event locations, the weighting values $W_{x}$ and $W_{m}$ would be added in the inversion. We test a lot of weighting values and find that it can improve the inverted results to set $W_{x}$ equals to 1000 and $W_{m}$ with respect to $\varepsilon, \delta$ and $\gamma$ equal to 1000. If $W_{x}$ equals to zero, the inversion is adopted to invert for only model parameters and the event location remains unchanged.

The non-linear inversion has a strong dependence on initial models. Under most circumstances, because of severe nonlinearity and trade-off, numerical iterations are needed but still cannot solve the problems, the test results will be shown in the following section. We suggest that slowness components of VSP data can be used to constrain the inversion above.

\section{Vertical slowness constrained traveltime inversion method (VTM)}

VSP surveys with multicomponent geophones provide a unique opportunity to measure the slowness components or polarization vectors. These measurements are employed in the inversion for the elements of the stiffness tensors, or the equivalent anisotropy parameters (Tsvankin and Grechka 2011; Tamimi et al., 2015). Although we can use polarization measurements, Asgharzadeh et al., (2015) suggest that for lateral homogeneous formation the slowness method is more accurate. 


\section{Non-peer reviewed EarthArXiv preprint}

Similar to the equations (5), the linear approximation of the vertical phase slowness components can be expressed to the perturbations of the group velocity model parameters,

For quasi-P wave, the linearized expression can be written as,

$$
\Delta P_{Z}^{q P}=\sum_{l=1}^{\text {raypath }} \frac{\partial P_{Z}^{q P}}{\partial \alpha_{0}^{l}} \Delta \alpha_{0}^{l}+\sum_{l=1}^{\text {raypath }} \frac{\partial P_{Z}^{q P}}{\partial \beta_{0}^{l}} \Delta \beta_{0}^{l}+\sum_{l=1}^{\text {raypath }} \frac{\partial P_{Z}^{q P}}{\partial \varepsilon^{l}} \Delta \varepsilon^{l}+\sum_{l=1}^{\text {raypath }} \frac{\partial P_{Z}^{q P}}{\partial \delta^{l}} \Delta \delta^{l}
$$

For quasi-Sv wave, the linearized expression can be written as,

$$
\Delta P_{Z}^{q S v}=\sum_{l=1}^{\text {raypath }} \frac{\partial P_{Z}^{q S v}}{\partial \alpha_{0}^{l}} \Delta \alpha_{0}^{l}+\sum_{l=1}^{\text {raypath }} \frac{\partial P_{Z}^{q S v}}{\partial \beta_{0}^{l}} \Delta \beta_{0}^{l}+\sum_{l=1}^{\text {raypath }} \frac{\partial P_{Z}^{q S v}}{\partial \varepsilon^{l}} \Delta \varepsilon^{l}+\sum_{l=1}^{\text {raypath }} \frac{\partial P_{Z}^{q S v}}{\partial \delta^{l}} \Delta \delta^{l}
$$

For SH wave, the linearized expression can be written as,

$\Delta P_{Z}^{S H}=\sum_{l=1}^{\text {raypath }} \frac{\partial P_{Z}^{S H}}{\partial \beta_{0}^{l}} \Delta \beta_{0}^{l}+\sum_{l=1}^{\text {raypath }} \frac{\partial P_{Z}^{S H}}{\partial \gamma^{l}} \Delta \gamma^{l}$

where $\Delta P_{Z}$ is the difference of theoretical and real vertical phase slowness components. The real components can be obtained by the adjacent receivers. We can extract the high-resolution arrival time difference between two receivers by crosscorrelating their recordings. Then the vertical slowness components can be approximately calculated by the arrival time difference dividing with their distance (Miller and Spencer, 1994), which is expressed as the equation (14). From the above equations, we can find that the slowness components are not dependent on the source parameters. Hence, we can extend the active source gather (Miller and Spencer, 1994) to common passive source gather (see Figure 1a), the source can be perforation shot or microseismic event with unknown source information. Relying on the above equations, the local anisotropic parameters can be derived effectively (Asgharzadeh et al., 2015). Therefore, the low-cost information can be applied to constrain the traveltime inversion. 
Therefore, the equation (9) can be further expressed as,

$$
\begin{aligned}
& \left(\begin{array}{ccc}
1 & W_{m} A_{T, m}^{q P} & W_{x} A_{x}^{q P} \\
1 & W_{m} A_{T, m}^{S H} & W_{x} A_{x}^{S H} \\
0 & W_{m} A_{P_{Z}, m}^{q P} & 0 \\
0 & W_{m} A_{P_{Z}, m}^{S H} & 0
\end{array}\right)\left(\begin{array}{c}
\Delta t_{0} \\
\Delta m / W_{m} \\
\Delta x / W_{x}
\end{array}\right)=\left(\begin{array}{c}
T_{o b s}^{q P}-T_{s y n}^{q P}-T_{0}^{q P} \\
T_{o b s}^{S H}-T_{s y n}^{S H}-T_{0}^{S H} \\
P_{Z, o b s}^{q P}-P_{Z, s y n}^{q P} \\
P_{Z, o b s}^{S H}-P_{Z, s y n}^{S H}
\end{array}\right) \\
& P_{Z} \approx\left(T_{i, k}-T_{j, k}\right) / d_{i j}
\end{aligned}
$$

In the inversion, we add a weighting scale to balance the arrival times and the vertical phase slowness components. The weighting value depends on the variation of the horizontal layer. If the layer could not be regarded as the homogeneous formation, we set the weighting to a small value. Additionally, in our inversion, only when two and two more receivers in the same layer, they can be utilized to calculate the vertical phase slowness components. If one layer contains no receivers or only one receiver, then the parameters of the layer will be not constrained in the above inversion. Hence, more receivers deployed in the same layer, better the layer's parameters are constrained; meanwhile, if the receivers can cover more layers, the inversion can constrain the inversion better.

\section{NUMERICAL EXAMPLES}

We construct a VTI layer model (Figure 2), which contains eight layers with one lowvelocity thin layer in the range from $1700 \mathrm{~m}$ to $2200 \mathrm{~m}$. To verify the validity of the vertical slowness estimations, in the model we randomly select 10 positions at the depth of $2 \mathrm{~km}$. Provided with ten horizontal slowness, ten vertical slowness components are derived using arrival time residual and distance of two adjacent receivers in the first 


\section{Non-peer reviewed EarthArXiv preprint}

layer. As shown in Figure 1a, the vertical slowness component is approximately calculated by the traveltime residual and distance of two neighboring receivers. Then we project ten slowness components on the theoretical slowness surface obtained from the Christoffel equation. Figure $1 \mathrm{~b}$ illustrates that these values are consistent with the theoretical values, which means these data are effective to be used to derive the model parameters.

To validate the efficiency of our method, we conduct four synthetic tests in our study. In the first two cases, the location is not considered in the inversion, that is, the inversion is only applied to yield model parameters and unknown origin time. In the case one, three perforation shots are placed nearby the receiver array. In the inversion, we assume that the locations of perforation shots are known, but their origin times remain unknown. And only two among five initial anisotropic parameters $\alpha_{0}$ and $\beta_{0}$, can be obtained by sonic logs, while other three initial models are set to zero. These model parameters are then calibrated by perforation shots using TM and VTM. Only traveltime data are used into the inversion of $\mathrm{TM}$, and both traveltime and vertical slowness components data are input into the VTM. Note that only the layers with at least two receivers could be uitilized to estimate vertical slowness components to constrain model parameters of the layer. The inversion results (Figure 3) show that almost all parameters are recovered well. In this case, the ray coverage is enough to the TM so that the inversion is not trapped in the local minimum and produces a satisfactory results. To test whether raypath coverage has an effect on the inversion, we shift the perforation shots to the right a lot. In the case two, the models conducted are the same 


\section{Non-peer reviewed EarthArXiv preprint}

to that of case one. The only difference is the positions of the perforation shots. The comparison of the results (Figure 5) using two different methods shows that VTM could still result in a stable results by the aid of the vertical slowness constraint while TM could not recover model parameters only using the traveltime information.

In some cases, if we only calibrate the model parameters by the perforation, it may cause velocity-related errors in locating the microseismic events, where the ray trajectories of the perforation shots do not cover (Grechka, 2010). Hence, we add three near-offset events into the case 3. In this case (Figure 6), the model parameters as well as three near-offset events are inverted simultaneously. The results of case three (Figure 7) illustrate that the location and model parameters are inverted well using both TM and VTM, because the added near-receiver events lead to a better raypath coverage. And the STM is also effective for the event location and model parameters recovery. If we add another three far-offset events into the case three, the location results (Figure 8) demonstrate that, for the locations results (marked by the purple color) of the TM, the near-offset event locations are better than the far-offset locations, but they all deviate from the true locations. However, the locations results of VTM demonstrate that all the event locations could be inverted well. Figure 9 illustrates that the VTM is more effective to recover the model parameters than TM. By the analysis we think that, once events with poor raypath coverage are put in the inversion, the results are hard to be recovered well, however, in the VTM the vertical slowness components could provide another constraint upon the traveltime inversion and make the inversion stable. In all synthetic cases, the rays cover the first six layers from the sources to the receivers, 
hence only the top six layers are updated in the inversion results. Additionally, in the fourth layer, due to its small thickness, the layer could not gain an effective ray coverage, and vertical slowness components are not measured in that layer, in which the Thomsen's parameters could not be therefore recovered well in both TM and VTM inversions.

\section{FIELD DATA EXAMPLE}

In this section, we apply two methods to a real data set, which is acquired from a single vertical monitoring well during a hydraulic fracturing treatment. Microseismic data were recorded during 12 stages of hydraulic fracturing. Twelve three-component receivers were deployed in a vertical monitoring well from $2070 \mathrm{~m}$ to $2415 \mathrm{~m}$ depth, with a receiver interval of $15 \mathrm{~m}$ (Figure 16). Within each stage, a ball-drop event was recorded with known location but an unknown origin time. And each ball-drop could trigger many microseismic events, which are mainly happened nearby the ball-drop. In addition, the sonic logging data could provide us a 1D velocity model but merely containing vertical P- and S-wave velocities. Under this circumstance, the vertical Pand S-wave velocities are regarded as the initial velocities in the inversion and other model parameters are all set to zero. In this study, we do not calibrate the model parameters using dropballs because the number of effective dropballs is limited. We select 37 events of stage 3 as well as 32 events of stage 4 during the processing. Figure 10 displays the recordings of a twelve-receiver array of one event, the arrivals of $\mathrm{P}$ and S waves can be clearly picked up. Upon the arrival times are archived, the vertical 


\section{Non-peer reviewed EarthArXiv preprint}

slowness data could be derived approximately using the equation (14). With these data, we apply TM and VTM to invert for the event locations and model parameters, respectively. Meanwhile, all events are located in a 2D profile and then projected to $3 \mathrm{D}$ by event azimuths from a separate analysis of the calibrated P-wave polarizations.

In the projections of location results (Figure 11), the event locations obtained with VTM are more convergent than the location results of TM, especially in the area marked by the green ellipse. Additionally, the $\mathrm{X}-\mathrm{Z}$ projection shows that the results of two stages are more separated obviously in VTM than in TM. Hence, the event location can be constrained better and more reasonable in VTM than in TM, which occurs because the vertical slowness components provide an additional constraint on the inversion process. In addition, the model results (Figure 12) with both inversion methods reveal weak anisotropy in the field situation, which can be observed in the recordings of the receiver array. Although we could not further verify the results in the field or with other supportive data, the above theoretical analysis and numerical examples help us understand the differences in the results of the two methods.

\section{CONCLUSION}

In this study, based on the traveltime inversion technique and the slowness method, we propose a method jointly using vertical slowness component and traveltime. This method can be applied to derive VTI parameters and event locations simultaneously or only calibrate the velocity model. Compared with the inversion solely using traveltime, our synthetic examples demonstrate that our method is more stable, especially with 


\section{Non-peer reviewed EarthArXiv preprint}

poor raypath coverage. In the first two synthetic examples, we do not invert for the event locations and only invert for the model parameters and unknown original times, the model parameters' recovery indicates that our method (VTM) is more stable and effective than the TM regardless of whether the raypath coverage is enough or not. In the last two synthetic cases, the event location as well as model parameters are inverted simultaneously in the inversion. Both cases show that vertical slowness components help the inversion constrain the event locations and model parameters well. In the field situation, the effectiveness of our method is significantly observed in the event locations. We note that, when the depth of event is larger than the receiver array, the approximation of vertical slowness is more accurate. In most real cases, the hydraulic fracturing usually sites deeper than receiver array installed in the borehole. Hence, our method is adaptive to be applied in the real example. Additionally, if the formation has a large variation of velocity, the slowness method is not effective. Under this circumstance, the slowness-polarization method can be utilized to handle the situation.

\section{ACKNOWLEDGMENTS}

We gratefully acknowledge the financial support of the National Natural Science Foundation of China (Grant No. 41374132). We appreciate the support from GeoTomo, allowing us to use MiVu software package to perform this study. 


\section{REFERENCES}

Alkhalifah, T., 2002, Traveltime computation with the linearized eikonal equation for anisotropic media, Geophysical Prospecting, 50(4), 373-382.

Asgharzadeh, M., Bona, A., Pevzner, R., Urosevic, M., Gurevich, B., 2012, Uncertainties in local anisotropy estimation from multi-offset VSP data, $74^{\text {th }}$ EAGE Conference and Exhibition incorporating EUROPEC.

Cerveny, V., 2005, Seismic ray theory, Cambridge university press.

Chapman, C., 2004, Fundamentals of seismic wave propagation, Cambridge university press.

Chapman, C.H., Miller, D.E., 1996, Velocity sensitivity in transversely isotropic media, Geophysical Prospecting, 44, 525-549.

Dewangan, P., Grechka, V., 2003, Inversion of multicomponent, multiazimuth, walkaway VSP data for the stiffness tensor, Geophysics, 68(3), 1022-1031.

De Parscau, J., 1991, Relationship between phase velocities and polarization in transversely isotropic media, Geophysics, 56(10), 1578-1583.

Erwemi, A., Walsh, J., Bennett, L., Woerpel, C., Purcell, D., 2010, Anisotropic Velocity Modeling For Microseismic Processing: Part 3-borehole Sonic Calibration Case Study, 80 ${ }^{\text {th }}$ SEG Annual Meeting, Expanded Abstracts, 508-512.

Eisner, L., Duncan, P.M., Heigl, W.M., Keller, W.R., 2009, Uncertainties in passive seismic monitoring, The Leading Edge, 28, 648-655.

Gaiser, J.E., 1990, Transversely isotropic phase velocity analysis from slowness 
estimates, Journal of Geophysical Research, Solid Earth, 95(B7), 11241-11254.

Grechka, V., Mateeva, A., 2007, Inversion of P-wave VSP data for local anisotropy: Theory and case study, Geophysics, 72(4), D69-D79.

Grechka, V., Mateeva, A., Gentry, C., Jorgensen, P., Lopez, J., Franco, G., 2007, Estimation of seismic anisotropy from P-wave VSP data, The Leading Edge, 26(6), 756-759.

Grechka, V., 2010, Data-acquisition design for microseismic monitoring. The Leading Edge, 29(3), 278-282.

Grechka, V., Singh, P., Das, I., 2011, Estimation of effective anisotropy simultaneously with locations of microseismic events, Geophysics, 76, WC141-WC153.

Han, S., 2016, A fast sweeping method for calculating qP traveltimes in TTI media \& joint microseismic events and anisotropic parameters inversion, PH.D. thesis, Univ. Sci and Tech. China (USTC).

Han, S., Zhang, W., Zhang, J., 2015, Joint Microseismic Quasi-P and SH Traveltime Inversion for Updating VTI Parameters, $85^{\text {th }}$ SEG Annual Meeting, Expanded Abstracts.

Horne, S., Leaney, S., 2000, Short note: Polarization and slowness component inversion for TI anisotropy, Geophysical Prospecting, 48(4), 779-788.

Kim, S., 1999, On eikonal solvers for anisotropic traveltimes, Geophysics, 64, $1867-$ 1876.

Li, J.L., 2013, Study of induced seismicity for reservoir characterization, PH.D. thesis, Mass. Inst. Tech. 
Li, J.L., Zhang, H., Rodi, W.L., Toksoz, M.N., 2013, Joint microseismic location and anisotropic tomography using differential arrival times and differential backazimuths, Geophys. J. Int., 195, 1971-1931.

Li J.L., Li, C., Morton, S.A., Dohmen, T., Katahara, K., Toksöz, M.N., 2014, Microseismic joint location and anisotropic velocity inversion for hydraulic fracturing in a tight Bakken reservoir, Geophysics, 79, 111-122.

Mah, M., Schmitt, D.R., 2003, Determination of the complete elastic stiffnesses from ultrasonic phase velocity measurements, Journal of Geophysical Research: Solid Earth, 108(B1).

Maxwell, S.C., Rutledge, J., Jones, R., Fehler, M., 2010, Petroleum reservoir characterization using downhole microseismic monitoring, Geophysics, 75, A129A137.

Maxwell, S.C., 2010, Microseismic: Growth born from success, The Leading Edge, 29, $338-343$.

Michel, O.J., Tsvankin, I., 2016, Anisotropic waveform inversion for microseismic velocity analysis and event location, 86 ${ }^{\text {th }}$ SEG Technical Program Expanded Abstracts, 296-300.

Miller, D.E., Spencer, C., 1994, An exact inversion for anisotropic moduli from phase slowness data, Journal of Geophysical Research: Solid Earth, 99(B11), 2165121657.

Pevzner, R., Gurevich, B., Urosevic, M., 2011, Estimation of azimuthal anisotropy from VSP data using multicomponent S-wave velocity analysis, Geophysics, 76(5), D1- 


\section{Non-peer reviewed EarthArXiv preprint}

D9.

Rusmanugroho, H., McMechan, G.A., 2012, Sensitivity of estimated elastic moduli to completeness of wave type, measurement type, and illumination apertures at a receiver in multicomponent VSP data, Geophysics, 77(1), R1-R18.

Rusmanugroho, H., McMechan, G.A., 2012, 3D, 9C seismic modeling and inversion of Weyburn Field data, Geophysics, 77(4), R161-R173.

Tang, W., Li, L., 2008, Exact traveltime computation in multi-layered transversely isotropic media with vertical symmetry axis, Acta Seismologica Sinica, 21, 370379.

Thomsen, L., 1986, Weak elastic anisotropy, Geophysics, 51, 1954-1966.

Tamimi, N., Tsvankin, I., Davis, T.L., 2015, Estimation of VTI parameters using slowness-polarization inversion of P-and SV-waves, Journal of seismic exploration, $24(5), 455-474$.

Walsh, J., Sinha, B., Plona, T., Miller, D., Bentley, D., Ammerman, M., 2007, Derivation of anisotropy parameters in a shale using borehole sonic data, $77^{\text {th }}$ SEG Annual Meeting, Expanded Abstracts, 323-327.

Warpinski, N.R., Waltman, C.K., Du, J., Ma, Q., 2009, Anisotropy effects in microseismic monitoring, Society of Petroleum Engineers.

Woerpel, C., 2010, Anisotropic velocity modeling for microseismic processing: Part 2fast and accurate model calibration with a cross-well source, $80^{\text {th }}$ SEG Annual Meeting, Expanded Abstracts, 508-512.

Zhang, H.J., Thurber, C.H., 2003, Double-difference tomography: the method and its 
Non-peer reviewed EarthArXiv preprint

application to the Hayward Fault, California, Bulletin of Seismological Society of America, 93, 1875-1889.

Zhang, J., Liu, H., Zou, Z., Huang, Z., 2015, Velocity modeling and inversion techniques for locating microseismic events in unconventional reservoirs, Journal of Earth Science, 26(4), 495-501.

Zhou, B., Greenhalgh, S.A., 2005, Analytic expressions for the velocity sensitivity to the elastic moduli for the most general anisotropic media, Geophysical Prospecting, $53,619-641$. 
(a)

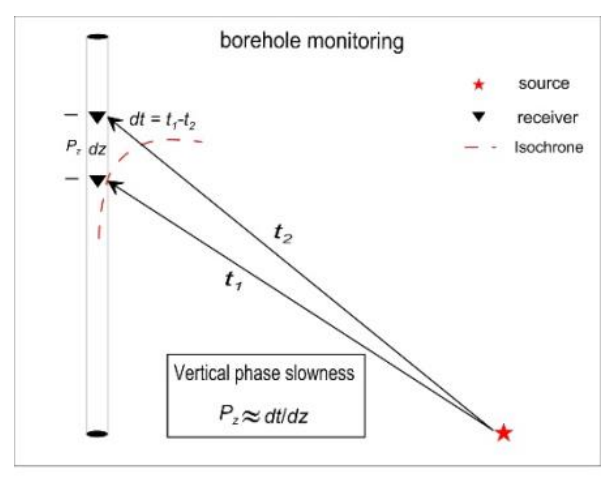

(b)

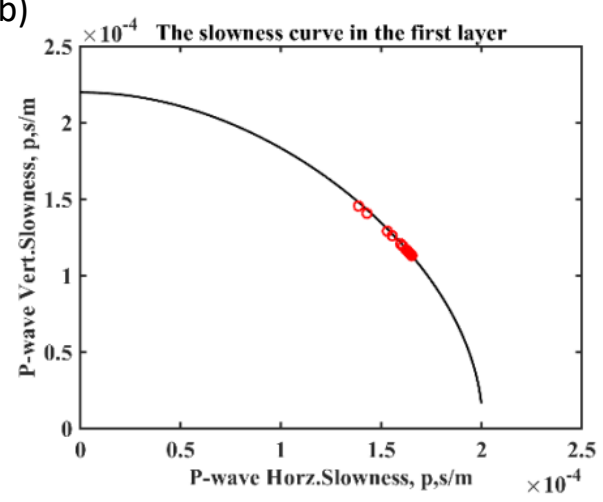

Figure 1 (a) the diagram of the slowness method; (b) the projection of the vertical slowness estimated using two receivers in the first layer (Figure 2) on the theoretical slowness surface. The red circles are from the ten samples at a depth of $2000 \mathrm{~m}$ in Figure 2. 
Non-peer reviewed EarthArXiv preprint

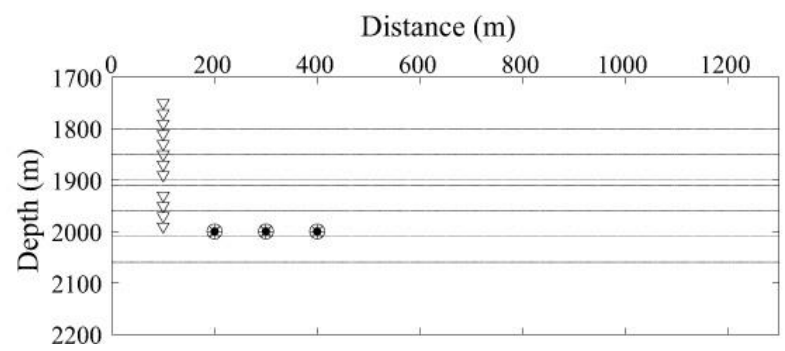

Figure 2 The survey geometry in case 1 . The velocity model includes eight layers from depth of $1700 \mathrm{~m}$ to $2200 \mathrm{~m}$. Twelve receivers in several layers are marked by triangles. Three circles represent perforations or drop-balls at known positions. Case 1 is an example with three near-offset perforations or drop-balls. 
Non-peer reviewed EarthArXiv preprint
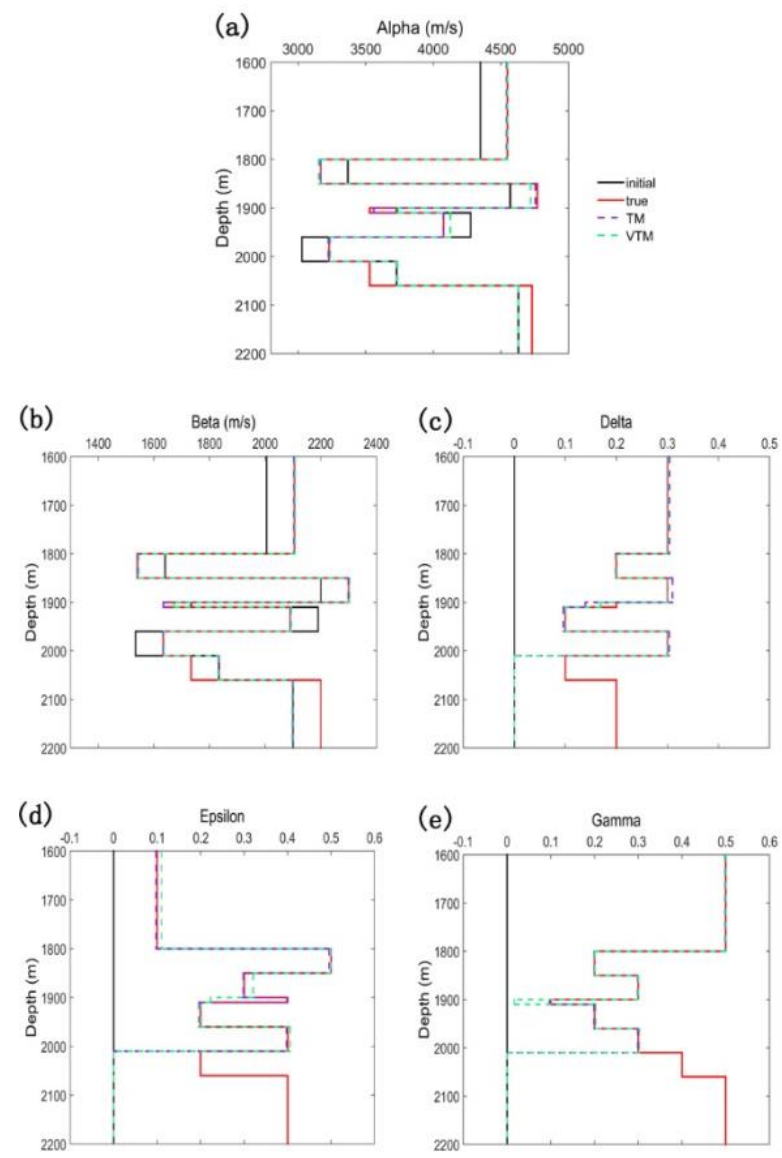

Figure 3 The inverted model results of case 1. (a, b, c, d, e) the five velocity solutions are derived by two methods. The black, red, purple, and green lines included stand for initial, true, and inverted parameters by TM and VTM, respectively. 
Non-peer reviewed EarthArXiv preprint

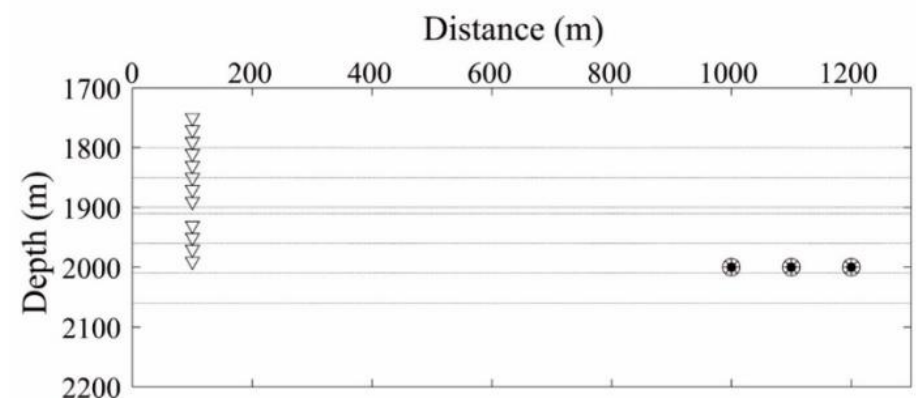

Figure 4 The survey geometry in case 2 . The model includes eight layers from depth of $1700 \mathrm{~m}$ to $2200 \mathrm{~m}$. Twelve receivers are marked by triangles, which are distributed in different layers. The three circles, black dots, and stars represent perforations or dropballs with known positions (they are fixed). Case 2 is the example with three far-offset perforations or drop-balls. 
Non-peer reviewed EarthArXiv preprint
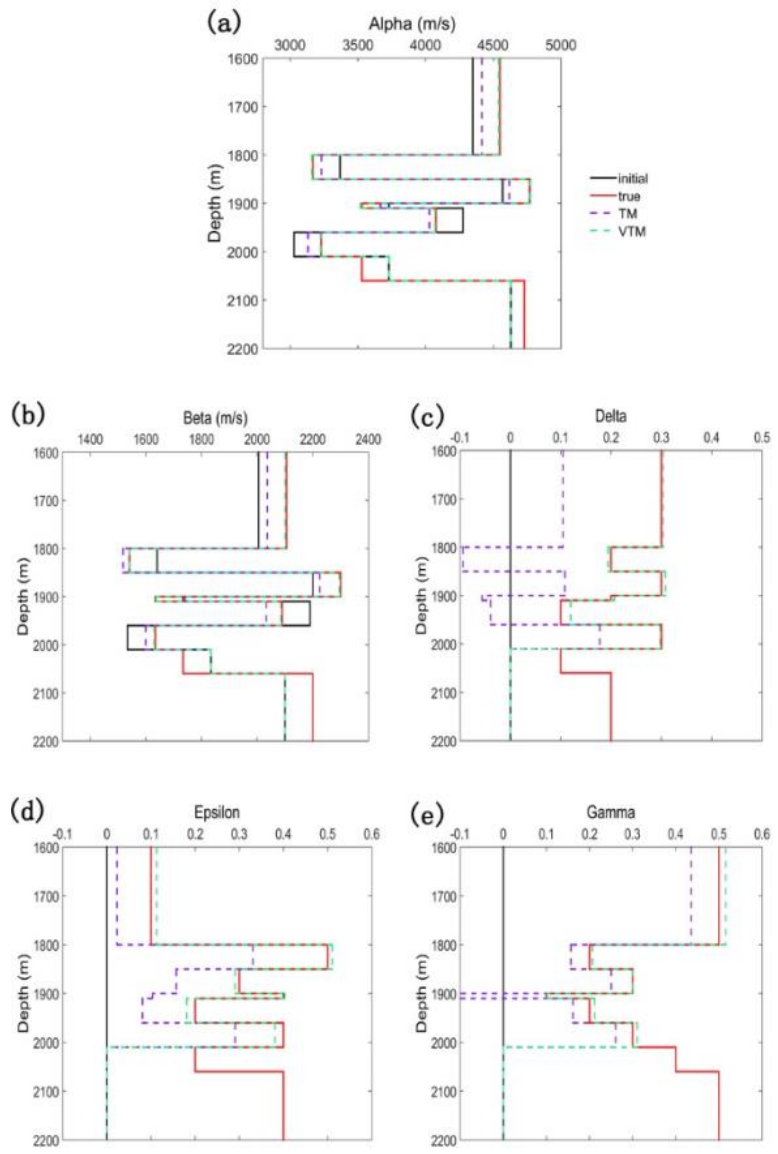

Figure 5 The inverted model results of case 2. (a, b, c, d, e) the five velocity solutions are derived by two methods. The black, red, purple, and green lines included stand for initial, true, and inverted parameters by TM and VTM, respectively. 
Non-peer reviewed EarthArXiv preprint

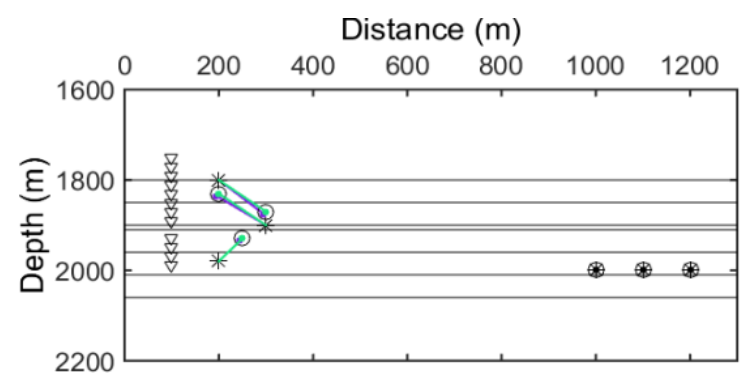

Figure 6 The model with survey geometry and traveltime misfit curves of case 3 . In the model the reverse triangles are the receivers, the perforation shots are marked by red rectangle and three stars, circles, and dots present the initial, true, and inverted locations, respectively. The purple and green represent TM and VTM method, respectively. 
Non-peer reviewed EarthArXiv preprint
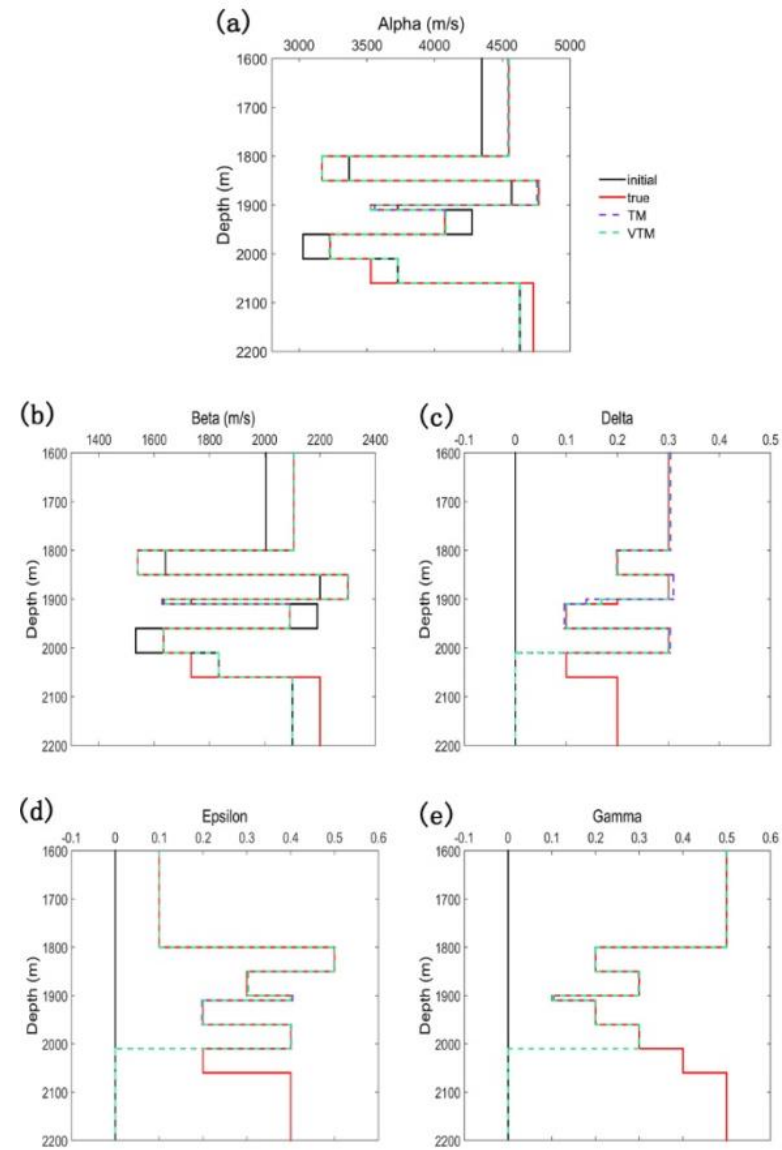

Figure 7 The inverted model results of case 3. (a, b, c, d, e) the five velocity solutions are derived by two methods. The black, red, purple, and green lines included stand for initial, true, and inverted parameters by TM and VTM, respectively. 
Non-peer reviewed EarthArXiv preprint

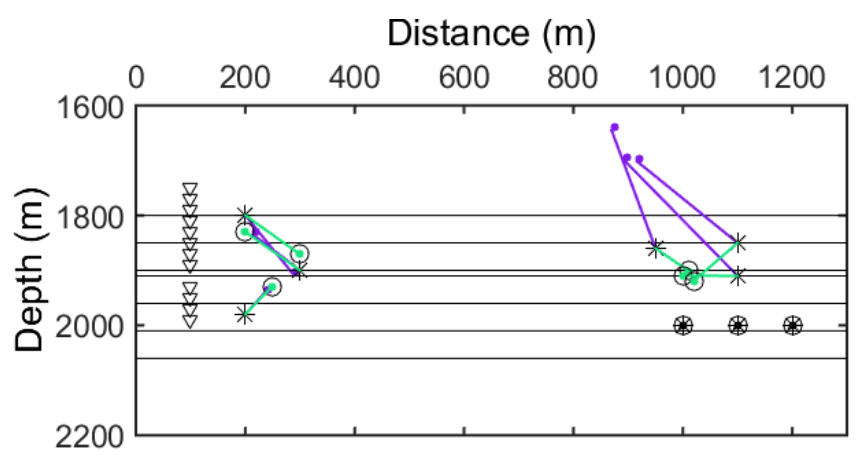

Figure 8 The model with survey geometry and traveltime misfit curves of case 4. (a) In the model the reverse triangles are the receivers, the perforation shots are marked by red rectangle and three stars, circles, and dots present the initial, true, and inverted locations, respectively. The purple and green represent TM and VTM method, respectively. 

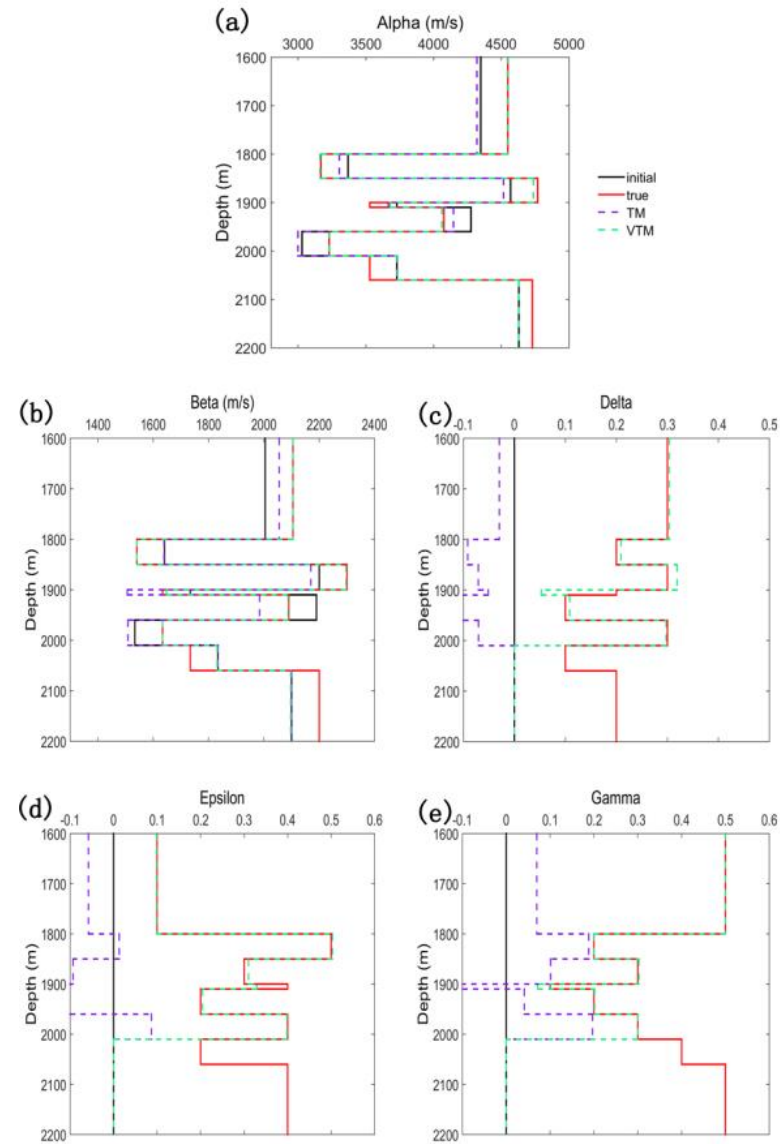

Figure 9 The inverted model results of case 4. (a, b, c, d, e) the five velocity solutions are derived by two methods. The black, red, purple, and green lines included stand for initial, true, and inverted parameters by TM and VTM, respectively. 
Non-peer reviewed EarthArXiv preprint

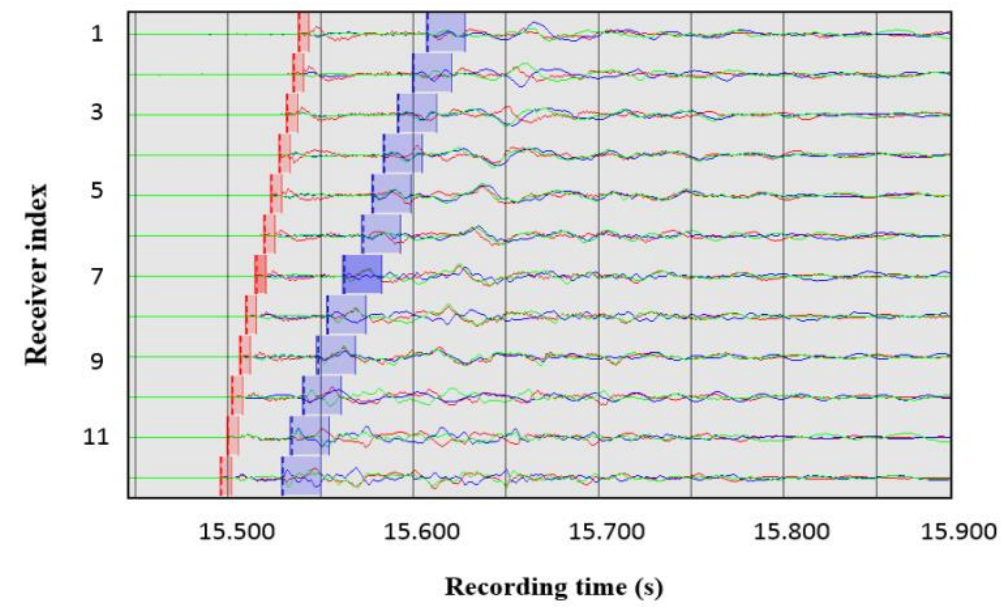

Figure 10 The waveform move-out for an event recorded by the vertical 12 receiver array during hydraulic stimulation. The P- and S- arrivals display a high SNR and are easily picked (red and blue respectively) on all 12 receivers. 

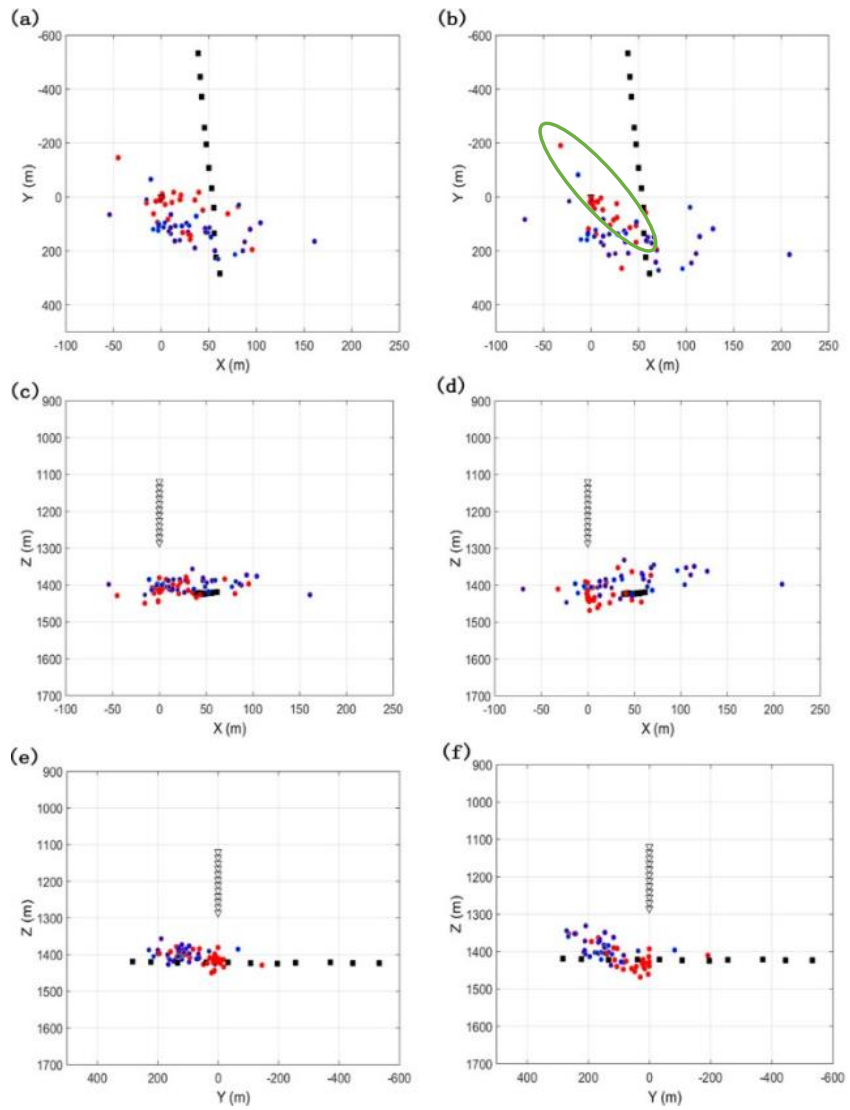

Figure 11 The different projections of event location results. The left maps (a, c, e) show the projections of locations on X-Y, X-Z, and Y-Z using VTM, and the right ones $(b, d, f)$ are the projections of locations on $X-Y, X-Z$, and $Y-Z$ using TM in isotropic media. The triangle array represents receiver array, the black squares are ball-drops. The red dots are the event location results of stage 3 , the blue ones are the event location results of stage 4 . 
Non-peer reviewed EarthArXiv preprint
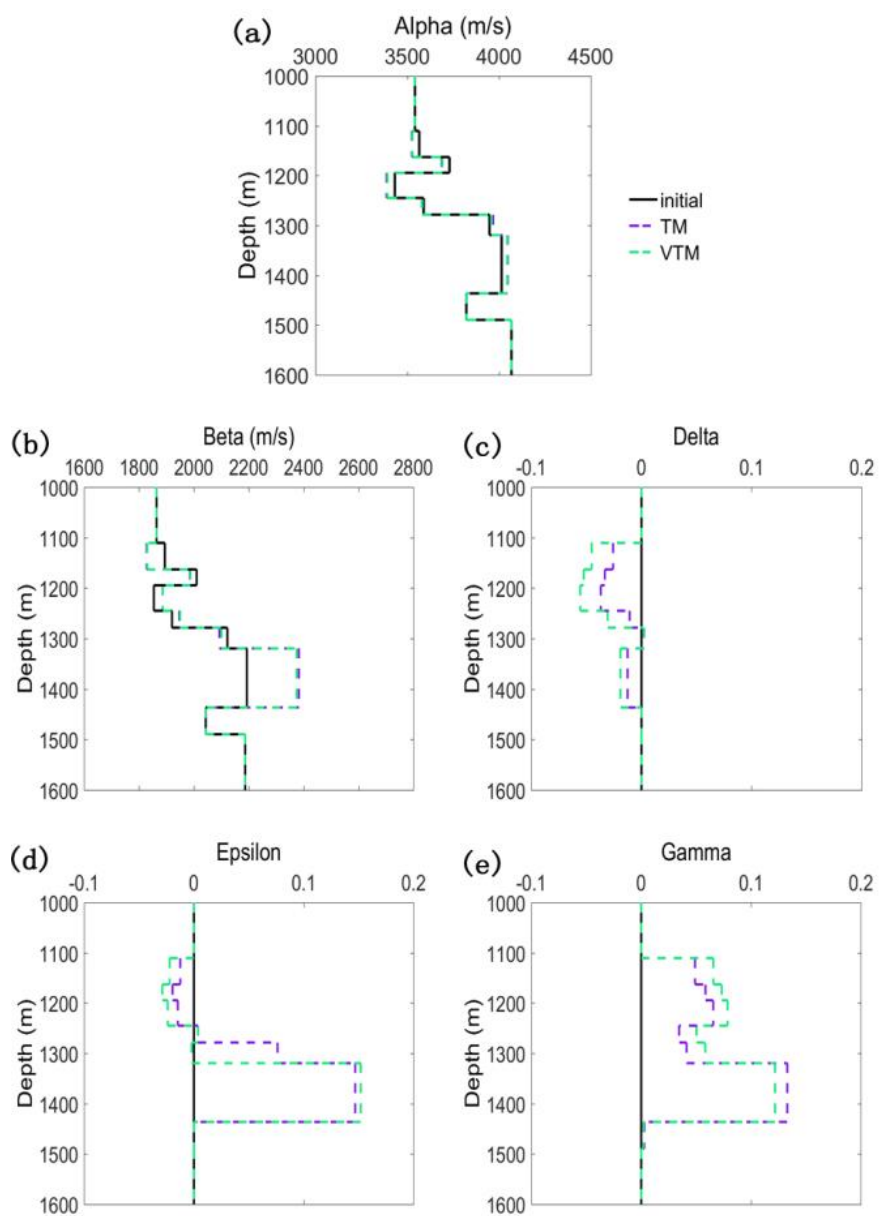

Figure 12 The inverted model results of the real case. (a, b, c, d, e) the five velocity solutions are derived by two methods. The black, red, purple, and green lines included stand for initial, true, and inverted parameters by TM and VTM, respectively. 